\title{
Extratos vegetais usados no controle de dípteros vetores de zoonoses
}

\author{
MACIEL, M.V. ${ }^{1 *}$ : MORAIS, S.M. ${ }^{2}$; BEVILAQUA, C.M.L.'; AMÓRA, S.S.A. ${ }^{1}$ \\ ${ }^{1}$ Universidade Estadual do Ceará, Laboratório de Doenças Parasitárias, Av. Paranjana, 1700, CEP: 60740-903, \\ Fortaleza-Brasil * michellinevet@gmail.com ${ }^{2}$ Universidade Estadual do Ceará, Laboratório de Química em \\ Produtos Naturais, Av. Paranjana, 1700, CEP: 60740-903, Fortaleza-Brasil
}

\begin{abstract}
RESUMO: Os insetos são importantes transmissores de doenças que cada vez mais ocorrem em áreas periurbanas ou urbanas, devido à introdução ou reaparecimento de seus respectivos vetores. As estratégias de controle das doenças transmissíveis por vetores biológicos são de difícil execução, principalmente quando associadas à existência de reservatórios domésticos e silvestres e aos aspectos ambientais. O uso de inseticidas direcionado ao combate de formas adultas de dípteros vetores tem sido freqüente. Entretanto, o amplo uso dos inseticidas sintéticos desde a descoberta do DDT para o controle de pragas domésticas e da agricultura, como também de vetores que transmitem doenças ao homem, levou a uma maior preocupação em relação à toxicidade e impacto ambiental destes agentes. Além disso, a resistência a inseticidas tornouse uma preocupação crescente na agricultura, economia e na saúde pública. Um grande número de diferentes espécies de plantas representando diferentes áreas geográficas ao redor do mundo tem se mostrado capaz de causar efeitos letais e subletais sobre insetos. Esta revisão aborda a utilização de extratos de plantas com potencial no controle de dípteros vetores de zoonoses.
\end{abstract}

Palavras-chave: Diptera, fitoquímicos, terpenos, alcalóides, substância deterrente

\begin{abstract}
Plant extracts used in the control of dipteran vectors of zoonosis. Insects are important transmitters of diseases increasingly occurring in peri-urban or urban areas due to the introduction or the reemergence of their respective vectors. Strategies to control diseases transmitted by biological vectors are difficult to implement, especially when associated with the existence of domestic and wild reservoirs and environmental aspects. The use of insecticides against adult forms of dipteran vectors has been frequent. However, the widespread use of synthetic insecticides since the discovery of DDT to control domestic and agricultural pests, as well as vectors that transmit diseases to humans, has led to greater concern for the toxicity and environmental impact of these agents. Moreover, resistance to insecticides has become an increasing concern in agriculture, economy and public health. A large number of different plant species representing different geographical areas around the world have been capable of causing lethal and sublethal effects on insects. This review addresses the use of potential plant extracts to control dipteran vectors of zoonosis.
\end{abstract}

Key words: Diptera, phytochemicals, terpenes, alkaloids, deterrent substance

\section{INTRODUÇÃO}

Os mosquitos transmitem doenças para aproximadamente 700 milhões de pessoas anualmente (Fradin \& Day, 2002). Existem mais de 3.000 espécies de mosquitos descritas, embora ainda se desconheça a biologia da grande maioria delas e os meios para combater aqueles prejudiciais ao homem (Consoli \& Lourenço de Oliveira, 1994). Algumas doenças passaram a ser transmitidas em áreas periurbanas ou urbanas, graças à introdução ou re-emergência de seus vetores nessas áreas. Atualmente no Brasil, as principais doenças vetoriais sujeitas a controle são dengue, malária, leishmanioses, doença de Chagas e as filarioses. Em razão da importância epidemiológica e do grau de participação do vetor no processo de transmissão, torna-se necessária a distinção nítida dos diferentes

Recebido para publicação em 23/07/2008

Aceito para publicação em 26/09/2009

Rev. Bras. PI. Med., Botucatu, v.12, n.1, p.105-112, 2010. 
tipos de vetores envolvidos na disseminação de doenças de vertebrados (Côrtes, 1993).

As estratégias de controle das doenças transmissíveis por vetores biológicos são complexas principalmente quando associadas à existência de reservatórios domésticos e silvestres e aos aspectos ambientais. Entretanto, o amplo uso dos inseticidas sintéticos desde a descoberta do DDT para o controle de pragas domésticas e da agricultura, como também de vetores que transmitem doenças ao homem, levou a maior preocupação em relação à toxicidade $\mathrm{e}$ impacto ambiental (Mulla \& Tianyun, 1999). Os principais problemas quando ocorre suspeita de resistência são o emprego de maiores concentrações ou quantidade do composto, na tentativa de recuperar a eficácia e o aumento da freqüência de aplicação e substituição do produto. Além de essas ações serem ambientalmente negativas, não são eficazes no controle da expansão territorial dos insetos vetores (Barreto, 2005). A existência de vetores resistentes a determinado produto químico pode ocorrer como resultado de fatores genéticos e operacionais, entre outros. A resistência é uma característica genética que se insere numa população em função do uso de inseticidas.

A capacidade dos insetos de tolerar concentrações inicialmente letais promove redução gradual na eficácia dos inseticidas, até a completa ineficiência. Os fatores operacionais também desempenham importante papel na implantação da resistência. Estes estão relacionados ao uso de inseticidas (classe, formulação e concentração, método de aplicação, freqüência de tratamentos, etc.) e por isso, podem ser perfeitamente controlados, fato que, na verdade, não ocorre devido ao uso indiscriminado dos produtos (Carvalho et al., 2004). Além disso, o custo para o desenvolvimento de inseticidas químicos é alto e tem aumentado ao longo dos anos devido à necessidade de novas moléculas e formulações mais adequadas, o que tem feito crescer o interesse pela pesquisa de inseticidas alternativos (Almeida \& Batista Filho, 2001).

Metabólitos secundários de plantas são conhecidos pelas propriedades inseticidas e em muitos casos as plantas têm história de uso para remédios caseiros e ainda para matar ou repelir insetos (Broussalis et al., 1999). O incentivo às investigações sobre interações plantas/insetos nas últimas décadas desvenda o uso potencial dos metabólitos de plantas ou aleloquímicos como agentes para esta finalidade (Pavela, 2004). Fitoquímicos obtidos de plantas com potencial no controle de insetos podem ser usados como uma alternativa às substâncias sintéticas ou adicionados a outros inseticidas nos programas de controle de vetores. Dependendo do tipo de atividade estas substâncias podem ser usadas como inseticidas, levando à morte larvas e adultos, ou como repelentes para proteção pessoal (ICMR Bulletin, 2003). O Brasil apresenta enorme riqueza botânica, com 56 mil espécies de plantas das 256 mil existentes no mundo, possuindo grande potencial como fonte de compostos biologicamente ativos provenientes de plantas medicinais. A utilização de plantas ou os extratos com atividade inseticida tem aplicação importante na saúde pública e na agropecuária (Matias et al., 2002).

\section{Plantas com potencial no controle de mosquitos}

Durante os primeiros 50 anos do século XX, predominaram os produtos inseticidas naturais de origem orgânica e inorgânica (Viegas-Júnior, 2003). $\mathrm{Na}$ década de 1950, Maranhão (1954) relacionou cerca de 2.000 plantas com propriedades inseticidas, distribuídas em 170 famílias, com atividade tóxica para diversos insetos. Os inseticidas comerciais de origem vegetal foram obtidos, principalmente a partir de cinco famílias botânicas: Solonaceae, Compositae, Leguminosae, Chenopodiaceae e Liliaceae, das quais foram extraídos, respectivamente, a nicotina, piretro, timbó, heléboro e anabasina (Maranhão, 1954). Schumutterer (1990) citou as famílias Meliaceae, Asteraceae, Labiaceae, Aristolochiaceae e Annonaceae, como principais fontes de princípios ativos inseticidas. Sabe-se que as plantas, como organismos que co-evoluíram com insetos e outros microrganismos, são fontes naturais de substâncias inseticidas e estas por sua vez, são produzidas pelo vegetal em resposta a um ataque patogênico (Simas et al. 2004). Estas defesas são de natureza química, e normalmente, envolvem substâncias do metabolismo secundário, as quais são chamadas de fitotoxinas ou aleloquímicos (Pinto et al., 2002). A Tabela 1 apresenta as fontes vegetais, classes químicas ativas e princípios ativos de plantas inseticidas, segundo Matias et al. (2002).

O emprego de substâncias extraídas de plantas, na qualidade de inseticidas, tem inúmeras vantagens quando comparado aos sintéticos: os inseticidas naturais são obtidos de recursos renováveis e são rapidamente degradáveis; 0 desenvolvimento da resistência dos insetos a essas substâncias, compostas da associação de vários princípios ativos é processo lento; estes pesticidas são de fácil acesso e obtenção e não deixam resíduos em alimentos, além de apresentarem baixo custo de produção (Roel, 2001).

$\mathrm{Na}$ busca pelo desenvolvimento de novos agentes ativos baseados em produtos naturais, esforços são feitos para selecionar, isolar, e desenvolver fitoquímicos com atividade pesticida (Mulla \& Tianyun, 1999). Várias são as estratégias capazes de determinar a atividade de produtos de origem natural contra insetos. De uma maneira geral, a 
TABELA 1. Plantas com propriedades inseticidas, princípios ativos e classes químicas.

\begin{tabular}{lcc}
\hline Plantas & Princípios ativos & $\begin{array}{c}\text { Classe química } \\
\text { ativa }\end{array}$ \\
\hline Nicotiana tabacum & Normonicotina & Alcalóides nicotínicos \\
Chrysanthemum cinerariefolium & Piretrina I & Piretróides \\
Derris spp. & Piretrina II & Rotenóides \\
Quassia amara & Rotenona & Quassinóides \\
Stemona japonica & Quassina & Alcalóides \\
Azadirachta indica & Estemofolina & Limonóides \\
Cymbopogon nardus & Azadirachtina & Terpenoides \\
\hline
\end{tabular}

pesquisa inicia-se com extratos brutos de plantas preparados com diversos solventes, tais como hexano, diclorometano, acetato de etila, metanol e água. Em seguida, os extratos ativos são fracionados através de métodos cromatográficos e as frações obtidas são testadas novamente, repetindo-se 0 processo até a obtenção do(s) composto(s) ativo(s) (Shaalan et al.,2005).

Os extratos obtidos com solventes orgânicos contêm complexa mistura de compostos ativos. Se a concentração letal excepcionalmente baixa é detectada, o extrato pode ser fracionado para extrair o componente químico responsável pelo efeito. Frações isoladas do mesmo extrato podem ter diferentes atividades larvicidas, pois contêm diferentes fitoquímicos. No entanto, alguns compostos, quando testados de forma isolada, apresentam baixa eficácia, sugerindo efeito sinérgico entre as substâncias presentes no extrato, indicando que a associação destas substâncias é a responsável pela mortalidade obtida nos testes realizados (Shaalan et al., 2005). É importante ressaltar que, para o uso de inseticidas botânicos, diversos aspectos devem ser levados em consideração, como a extração, conservação dos extratos, dosagem eficiente, estabilidade, toxicidade e custo. Todos estes aspectos são avaliados quando se identifica as principais substâncias contidas no inseticida (Nogueira \& Palmério, 2001).
A escolha do bioensaio mais apropriado para determinar a atividade inseticida depende dos hábitos dos insetos a serem combatidos (Simões et al., 1998). Em termos gerais, larvas de três espécies de mosquitos de importância em saúde pública, pertencentes aos gêneros Aedes, Anopheles e Culex são utilizadas para triagem de fitoquímicos, devido a susceptibilidade em maior ou menor grau a estes compostos. A bioatividade de fitoquímico contra larvas de mosquitos pode variar significativamente dependendo da espécie, parte utilizada e idade da planta, bem como o solvente usado na extração (Shaalan et al., 2005). O uso de grupos homogêneos de larvas dos mosquitos para estudos de laboratório é de fundamental importância para determinar a atividade de compostos sintéticos, IGRs, inseticidas de origem bacteriana e produtos naturais (WHO, 2005). A seleção da espécie de mosquito também é de fundamental importância já que ocorrem variações dentro do próprio gênero e espécie (Shaalan et al. 2005).

Em geral, larvas de Aedes são mais robustas e menos susceptíveis a inseticidas e extratos botânicos do que as larvas de Culex, por exemplo. Já a susceptibilidade das larvas de Anopheles pode variar desde que elas podem ser mais ou menos susceptíveis que as larvas de Culex e Aedes aos derivados botânicos e inseticidas. Aedes aegyptié o 
inseto mais comumente usado para a triagem de substâncias com ação inseticida por ser menos susceptível e de fácil colonização em laboratório, (Shaalan et al. 2005) apresentam boa revisão sobre fitoquímicos como potenciais agentes para o controle de mosquitos. A Tabela 2 apresenta algumas plantase os respectivos efeitos sobre mosquitos vetores de zoonoses.

Dentre os testes realizados com fitoquímicos, os óleos essenciais de vegetais são os mais utilizados como atraentes ou repelentes de insetos (Bowman, 2006). Em muitas plantas são encontradas substâncias geralmente voláteis que podem ser detectadas pelas antenas ou tarsos de insetos. Entre essas, estão os monoterpenos (citronelal, linalol, mentol, pinenos, mentona, carvona e limoneno), os sesquiterpenos (farnesol, nerolidol), os fenilpropanóides (safrol, eugenol) e muitos outros compostos (Simões \& Sptizer, 2004). Muitas espécies de plantas da Família Lamiaceae são tóxicas para insetos, como as do gênero Ocimum spp (Palsson \& Jaenson, 1999). Extratos de plantas

TABELA2. Efeito de extratos de algumas plantas sobre mosquitos potenciais vetores de zoonoses.

\begin{tabular}{|c|c|c|c|c|}
\hline Plantas & $\begin{array}{l}\text { Óleo/extrato } \\
\text { /concentração }\end{array}$ & Inseto & Ação & Referência \\
\hline A. indica & $\begin{array}{l}\text { Óleo de sementes } \\
\text { (50ppm) }\end{array}$ & A. aegypti & $\begin{array}{c}\text { Inibição de } \\
\text { emergência de } \\
\text { insetos }\end{array}$ & Sinniah et al. 1994 \\
\hline Ocimum gratissimum & $\begin{array}{c}\text { Óleo essencial ( } \mathrm{CL}_{50} \\
60 \mathrm{ppm})\end{array}$ & & & \\
\hline Ocimum americanum & $\begin{array}{l}\text { Óleo essencial (CL50 } \\
67 \text { ppm) }\end{array}$ & A. aegypti & Mortalidade de larvas & $\begin{array}{r}\text { Cavalcanti et al. } \\
2004\end{array}$ \\
\hline Lippia sidoides & $\begin{array}{c}\text { Óleo essencial ( } \mathrm{CL}_{50} \\
63 \mathrm{ppm})\end{array}$ & & & \\
\hline Cymbopogon citratus & $\begin{array}{l}\text { Óleo essencial }\left(C_{50}\right. \\
69 \mathrm{ppm})\end{array}$ & & & \\
\hline Mentha piperita & $\begin{array}{l}\text { Óleo essencial } \\
\left(3 \mathrm{~mL} / \mathrm{m}^{2}\right)\end{array}$ & $\begin{array}{c}\text { A. aegypti } \\
\text { A. stephensi } \\
\text { Cx. quinquefasciatus } \\
\text { A. aegypti }\end{array}$ & $\begin{array}{l}100 \% \text { Mortalidade } \\
\text { larval }\end{array}$ & Ansari et al. 2000 \\
\hline Pinus longifolia & $\begin{array}{l}\text { Oleo essencial (200 } \\
\text { ppm) }\end{array}$ & $\begin{array}{c}\text { A. stephensi } \\
\text { Cx. quinquefasciatus }\end{array}$ & $\begin{array}{l}\text { 100\% Mortalidade } \\
\text { larval }\end{array}$ & Ansari et al. 2005 \\
\hline Apium graveolens & $\begin{array}{c}\text { Oleo essencial (120 } \\
\text { ppm) }\end{array}$ & A. aegypti & $\begin{array}{c}96,2 \% \text { de mortalidade } \\
\text { larval }\end{array}$ & $\begin{array}{r}\text { Choochote et al. } \\
2004\end{array}$ \\
\hline Ageratum conyzoides & $\begin{array}{l}\text { Oleo essencial }(0,5 \\
\text { ppm) }\end{array}$ & A. aegypti & $\begin{array}{l}100 \% \text { de mortalidade } \\
\text { larval }\end{array}$ & $\begin{array}{r}\text { Mendonça et al. } \\
2005\end{array}$ \\
\hline Croton zenhtneri & $\begin{array}{c}\text { Óleo essencial ( } \mathrm{CL}_{50} \\
28 \mathrm{ppm})\end{array}$ & & & \\
\hline Croton nepetaefolius & $\begin{array}{c}\text { Óleo essencial }\left(C_{50}\right. \\
84 \mathrm{ppm})\end{array}$ & A. aegypti & Mortalidade de larvas & Morais et al. 2006 \\
\hline $\begin{array}{l}\text { Croton } \\
\text { argyrophylloides }\end{array}$ & $\begin{array}{c}\text { Óleo essencial }\left(\mathrm{CL}_{50}\right. \\
102 \text { ppm) }\end{array}$ & & & \\
\hline Croton sonderianus & $\begin{array}{c}\text { Óleo essencial }\left(C_{50}\right. \\
104 \text { ppm) }\end{array}$ & & & \\
\hline $\begin{array}{l}\text { Copaifera langsdorff } \\
\text { Aegiphila ihotskiana }\end{array}$ & $\begin{array}{l}\text { Oleo essencial }(0,5 \\
\text { ppm) }\end{array}$ & A. aegypti & $\begin{array}{l}100 \% \text { de mortalidade } \\
\text { larval }\end{array}$ & $\begin{array}{r}\text { Mendonça et al. } \\
2005\end{array}$ \\
\hline Lippia sidoides & Óleo essencial puro & A. aegypti & $100 \%$ de mortalidade & Carvalho et al. 2003 \\
\hline $\begin{array}{l}\text { Rhinacanthus } \\
\text { nasutus }\end{array}$ & $\begin{array}{c}\text { Fração éter de } \\
\text { petróleo de folhas }\end{array}$ & An. Stephensi & $\begin{array}{c}\text { Diminuição de } 45 \% \text { na } \\
\text { eclosão de ovos }\end{array}$ & $\begin{array}{r}\text { Muthukrishman \& } \\
\text { Pushpalatha, } 2001\end{array}$ \\
\hline & & Cx. quinquefasciatus & $\begin{array}{c}\text { Diminuição de } 50 \% \text { na } \\
\text { eclosão de ovos }\end{array}$ & \\
\hline Melia azedarach & $\begin{array}{l}\text { Extrato metanólico } \\
\text { de sementes }(2 \%)\end{array}$ & A. stephensi & $\begin{array}{c}92 \text { e } 90 \% \text { de } \\
\text { mortalidade pupas e } \\
\text { adultos }\end{array}$ & Nathan et al. 2005 \\
\hline $\begin{array}{l}\text { Calophyllum } \\
\text { inophyllum }\end{array}$ & $\begin{array}{l}\text { Fração acetato de } \\
\text { etila de sementes } \\
\quad(0.33 \text { ppm })\end{array}$ & An. Stephensi & $\begin{array}{c}\text { Aumento de } 50 \% \text { no } \\
\text { do período de } \\
\text { desenvolvimento }\end{array}$ & $\begin{array}{r}\text { Muthukrishman et al. } \\
1999\end{array}$ \\
\hline
\end{tabular}

Rev. Bras. PI. Med., Botucatu, v.12, n.1, p.105-112, 2010. 
incluindo Ocimum basilicum, O. gratissimum, $O$. americanum, Cymbopogom nardus, Alpinia galanga, Syzyaium aromaticum e Thymus vulgaris, Mentha, Eucalyptus maculata citriodon, Tagetus e Lantana camara (Ansari et al. 2005) têm sido estudadas como possíveis repelentes de mosquitos. Os monoterpenos limoneno, terpinoleno, citronelol e citronelal e cânfora (Figura 1) são os mais comuns constituintes de alguns óleos que têm sido relatados com propriedades repelentes sobre vários insetos (Jantan \& Zaki, 1998).

Alguns fitoquímicos apresentam atividade deterrente. Uma substância deterrente é aquela que impede a alimentação do inseto, levando-o à morte. Esta ação já foi descrita para a rotenona (Simões,
1998). De acordo com Mordue (Luntz) e Nisbet (2000), a deterrência é um distúrbio que está associado a mecanismos sensoriais e causa redução do consumo de alimento, provocando deficiência nutricional. A falta de nutrientes, por sua vez, pode ocasionar atraso no desenvolvimento ou deformações. Da mesma forma, a ocorrência de deformações ou deficiência nutricional, diminui também a capacidade de movimentação do inseto, na procura por alimentos de melhor qualidade ou de locais para abrigo ou reprodução, tornando-o também mais suscetível ao ataque de inimigos naturais.

Os reguladores de crescimento de insetos formam um grupo de inseticidas de nova geração que causam modificações fisiológicas e morfológicas

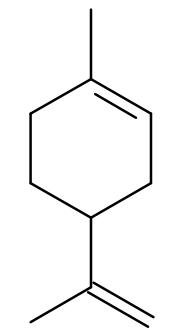

Limoneno

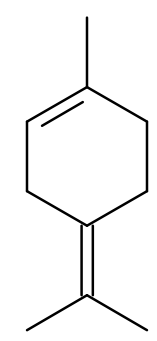

Terpinoleno

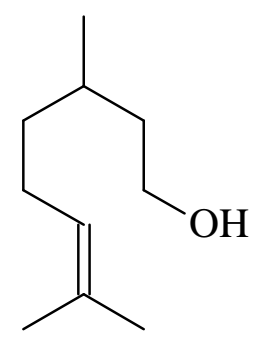

Citronelol

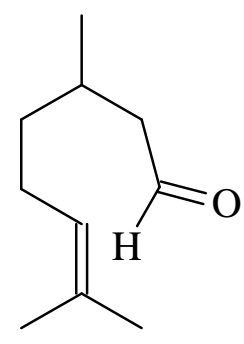

Citronelal

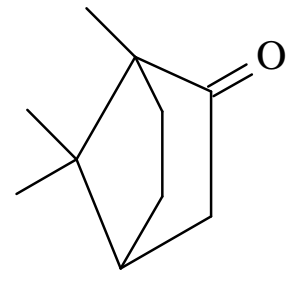

Cânfora

FIGURA 1: Estruturas químicas dos principais constituintes presentes em óleos essenciais repelentes de insetos.

durante o desenvolvimento do inseto. Este grupo é mais conhecido pela sigla IGR (Insect Growth Regulator). Os IGRs surgiram na década de 1970 como novo grupo de inseticidas de ação mais específica e menor toxicidade para os mamíferos do que os outros inseticidas pois atuam seletivamente ao interromper o desenvolvimento e crescimento ao invés de promover a intoxicação direta (Silva \& Mendes, 2002). Estes produtos são mais seguros e eficientes dentre os inseticidas disponíveis. A segurança reside no fato de que os hospedeiros mamíferos não possuem hormônios juvenis ou receptores destes hormônios. Extratos botânicos possuem substâncias reguladoras do crescimento que podem atuar sobre o período de desenvolvimento, crescimento, emergência de adultos, fecundidade, fertilidade e eclosão de ovos dos insetos (Shaalan et al. 2005). Os IGR mais utilizados no controle de mosquitos pertencem ao grupo das benzoil-feniluréias (BPU, inibidores de síntese de quitina) ou são compostos quimicamente relacionados ao hormônio juvenil natural de insetos, designados como análogos de hormônio juvenil (AHJ) (Slama et al., 1974 citado por Braga \& Valle, 2007). No Brasil, Coelho et al. (2006) adicionaram azadirachtina à dieta de larvas de Lu. longipalpis em laboratório, e verificaram que nas concentrações de $0,1 \mu \mathrm{g}$ de azadirachtina $\mathrm{mg}^{-1}$ de dieta, houve aumento significante da mortalidade larvar quando comparado ao grupo controle. A concentração de $10 \mu \mathrm{g}$ de azadirachtina $\mathrm{mg}^{-1}$ foi a que promoveu maior percentual de mortalidade das larvas $(74,4 \pm 8,3 \%)$. As concentrações de 0,1 e $1,0 \mu \mathrm{g}$ de azadirachtina $\mathrm{mg}^{-1}$ de dieta bloquearam a muda das larvas do inseto, que permaneceram no terceiro estágio até o final do experimento. Além disso, a adição simultânea de ecdisona ( $1 \mu \mathrm{g} \mathrm{mg}^{-1} /$ dieta) à dieta reverteu os efeitos inibidores desta substância. Concluiu-se que a azadirachtina é potente inibidor do crescimento de larvas Lu. longipalpis. Já os fagodeterrentes são substâncias naturais que têm a propriedade de interromper o repasto sangüíneo, podendo este efeito ser temporário ou permanente. Os fagorrepelentes geralmente agem sobre o sistema nervoso central dos insetos e são específicos para determinadas espécies (Pinto et al., 2002). Plantas da família Meliaceae são conhecidas por conter uma variedade de compostos com atividade inseticida, fagoinibidora, e reguladora do crescimento (Nakatani et al., 2004), estas últimas devido à presença dos limonóides (Nathan et al., 2005). Estes compostos podem ser encontrados em todos os tecidos das plantas desta família, no entanto, diversas partes das plantas podem individualmente produzir diferentes tipos de limonóides (Matias et al., 2002).

Existe grande diversidade de limonóides isolados da família Meliaceae, entre eles, as 
Existe grande diversidade de limonóides isolados da família Meliaceae, entre eles, as azedarachinas, sendaninas e trichilinas, além dos que apresentam o anel C-seco. Os limonóides com anel C-seco restringem-se aos gêneros Azadirachta e Melia sp. (Champagne et al., 1992). Estes compostos possuem o anel $\mathrm{C}$ do núcleo dos tetranotriterpenóides aberto como pode ser observado na azadiractina, que é o maior representante desta classe (Mulla \& Tianyun, 1999). A azadirachtina (Figura 2), triterpeno isolado de $A$. indica e $M$. azedarach é exemplo de fagorrepelente (Pinto et al., 2002). Mulla \& Tianyun (1999) relataram diversos trabalhos mostrando a atividade fagoinibidora de várias formulações do "neem" (nome popular de $A$. indica), sobre mosquitos dos gêneros Anopheles e Culex.

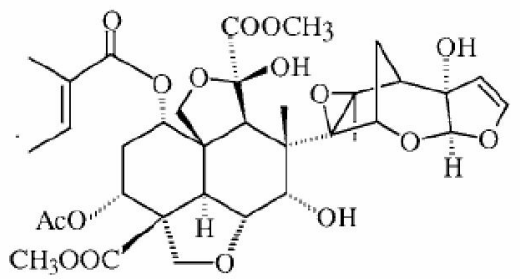

FIGURA2. Estrutura química da Azadirachtina (Isman, 2006).

Outras famílias de plantas apresentam substâncias fagorrepelentes. As famílias Papilionaceae, Loganiaceae e Fabaceae são ricas em rotenonas e rotenóides. A rotenona (Figura 3) é um produto inseticida obtido de raízes de diversas plantas (Simões, 1998). A rotenona paralisa o sistema respiratório de peixes trazendo-os à superfície, tem sido usada tradicionalmente na pesca de subsistência indígena em sistemas de água doce e marinho dessas áreas (Bearez, (1998), Lockett, (1998), Ling, (2003)). Esta substância é componente inseticida das raízes de Derris sp, planta pertencente ao gênero Lonchocarpus e de vários arbustos leguminosos (Bowman, 2006). Luitgards-Moura et al. (2002) utilizaram extratos aquosos do caule de Derris amazonica e folhas de Antonia ovata em experimentos com adultos de Lu. longipalis e verificaram que após 72 horas de exposição à $D$. amazônica houve um percentual de mortalidade de $100 \%$ das fêmeas destes insetos quando em concentração muito alta ( $\left.250 \mathrm{~g} \mathrm{~L}^{-1}\right)$. Os machos foram mais susceptíveis ( $80 \%$ de mortalidade) ao extrato das folhas de $A$. ovata na mesma concentração alta. Estes resultados dificultam (diminuem, desencorajam) as possibilidades da utilização dessas plantas no controle desse inseto vetor.

Um outro tipo de ação inseticida é através do bloqueio da neurotransmissão. Algumas substâncias inseticidas agem rapidamente paralisando e matando os artrópodes por interromperem o transporte de íons

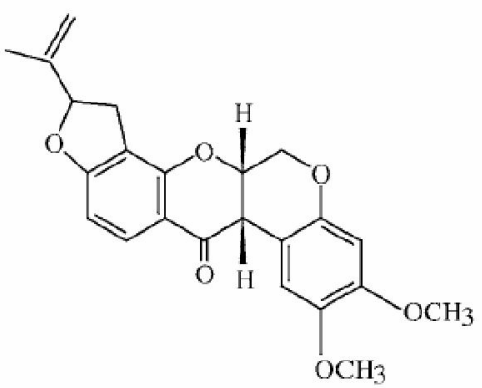

FIGURA 3. Estrutura química da Rotenona (Isman, 2006).

sódio e potássio nas membranas de células nervosas, bloqueando a neurotransmissão ao longo do axônio e da sinapse (Bowman, 2006). É o caso dos piretróides e piretrinas (Figura 4). Estas substâncias formam um grupo de inseticidas derivados de plantas da família Asteraceae já utilizados na antiga Pérsia. A ação sobre os insetos é caracterizada pela rápida morte, particularmente em insetos voadores, hiperatividade e convulsões. Desta forma, o mecanismo de ação destes inseticidas é qualitativamente similar ao DDT e outros organoclorados. Pyretrum refere-se ao óleo extraído das folhas secas de Tanacetum cinerariaefolium (Asteraceae) (Isman, 2006).

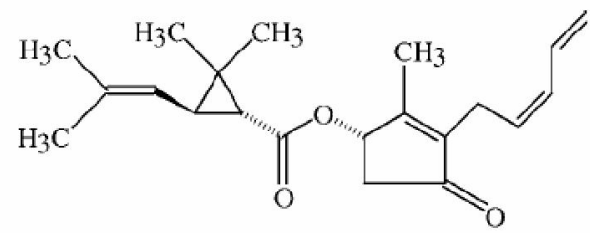

FIGURA 4. Estrutura química da piretrina I (Isman, 2006).

O flebotomíneo Phlebotomus papatasi transmite Leishmania major, que causa leishmaniose cutânea em vastas regiões do Velho Mundo. Além de sangue este mosquito também se alimenta de flores. Num estudo sobre dieta foi observado que uma noite de alimentação com Solanum jasminoides, Ricinus communis, ou Bougainvillea glabra diminuiu drasticamente o período de vida dos flebótomos. No entanto em regiões endêmicas para $L$. major em lugares de grande incidência do inseto, o número de $P$. papatasiem armadilhas próximas de $B$. glabra foi oito vezes menor (62 contra 502 mosquitos capturados) do que nos lugares não próximos (do controle). Estes resultados implicam que $B$. glabra garante proteção contra o inseto, reduzindo o risco de leishmaniose. Os autores sugerem que esta e outras plantas ornamentais são danosas para o flebotomíneo e podem ser úteis para reduzir o risco de leishmaniose (Schlein et al., 2001).

Um grande número de diferentes espécies 
de plantas representando diversas áreas geográficas ao redor do mundo tem apresentado compostos capazes de causar efeitos tóxicos e agudos sobre insetos (Shaalan et al. 2005). Entretanto, Isman (1997) relatou algumas barreiras para a comercialização destes fitoquímicos. Dentre estas, tem-se (1) a escassez de recursos botânicos, sendo necessária a produção de inseticidas botânicos em larga escala para a comercialização, a menos que a planta em questão seja extremamente abundante na natureza ou já tenha sido produzida para outras finalidades; (2) padronização dos extratos e controle de qualidade, baseado nos ingredientes ativos; e (3) dificuldades de registro destes fitoquímicos. Esta situação pode impedir muitos pesticidas botânicos de alcançar a esfera comercial em países onde existe grande demanda destes produtos. Apesar disso, a busca de novos inseticidas constitui-se um campo de investigação aberto, amplo e contínuo. A grande variedade de substâncias presentes na flora continua sendo enorme atrativo na área de controle de insetos, principalmente levando-se em consideração que apenas pequena parcela destas plantas foi investigada com tal finalidade (Simões \& Spitzer, 2004).

\section{REFERÊNCIA}

ALMEIDA, J.E.M.; BATISTA FILHO, A. Banco de microrganismos entomopatogênicos. Biotecnologia Ciência \& Desenvolvimento, v.21, p.30-3, 2001.

ANSARI, M.A. et al. Larvicidal and insect repellent activities of pine (Pinus longifolia, Family: Pinaceae) oil. Journal Veterinary Borne Disease, v.42, p.95-9, 2005.

ANSARI, M.A. et al. Larvicidal and repellent actions of Dalbergia sissoo Roxb. (F. Leguminosae) oil against mosquitoes. Bioresource Technology, v.73, p.207-11, 2000.

BARRETO, C.F. Aedes aegypti - Resistência aos Inseticidas Químicos e as Novas Alternativas de Controle. Revista Eletrônica Faculdade Montes Belos, v.1, p.6273, 2005.

BOWMAN, D.D. Parasitologia Veterinária de Georgis. 8.ed. Barueri: Manole, 2006. 422p.

BRAGA, I.A.; VALLE, D. Aedes aegypti: Inseticidas, mecanismo de ação e resistência. Epidemiologia e serviços de saúde, v.16, p.279-93, 2007.

BROUSSALIS, A.M. et al. Argentine plants as potential source of insecticidal compounds. Journal of Ethnopharmacology, v.67, p.219-23, 1999.

CARVALHO, A.F.U. et al. Larvicidal activity of the essencial oil from Lippia sidoides Cham against Aedes aegypti Linn. Memórias do Instituto Oswaldo Cruz, v.98, p.56971, 2003.

CARVALHO, M.S.L. et al. Suscetibilidade de larvas de Aedes aegypti ao inseticida temephos no Distrito Federal. Revista de Saúde Pública, v.38, p.623-9, 2004. CAVALCANTI, E.S.B. et al. Larvicidal activity of essencial oils from Brazilian plants against Aedes aegypti. Memórias do Instituto Oswaldo Cruz, v.99, p.541-4, 2004.
CHAMPAGNE, D.E. et al. Biological activity of limonoids from the Rutales. Phytochemistry, v.31, p.377-94, 1992. CHOOCHOTE, W. et al. Potential of crude extract of celery, Apium graveolens L., against the insect Aedes aegypti (L) (Diptera: Culicidae). Journal of Vector Ecology, v.29, p.340-6, 2004.

COELHO, A.A.A. et al. Effects of Azadirachtin on the development and mortality of Lutzomyia longipalpis larvae (Diptera: Psychodidae: Phlebotominae). Jornal of Medical Entomology, v.43, p.262-6, 2006.

CONSOLI, R.A.G.B.; LOURENÇO DE OLIVEIRA, R. Principais mosquitos de importância sanitária no Brasil. Rio de Janeiro: Fiocruz, 1994. 228p.

CÔRTES, J.A. Epidemiologia conceitos e princípios fundamentais. São Paulo: Livraria Varela, 1993. 286p. FRADIN, M.S.; DAY, J.F. Comparative efficacy of insect repellents against mosquito bites. The New England Journal of Medicine, v.347, p.13-8, 2002.

ICMR BULLETIN. Prospects of using products in the control of insect vectors, v.33, p.1-10, 2003.

ISMAN, M.B. Botanical Insecticides, deterrents, and repellents in modern agriculture and an increasingly regulated world. Annual Review Entomology, v.51, p.4566, 2006.

ISMAN, M.B. Neem and other botanical insecticides: Barriers to commercialization. Phytoparasitica, v.25, p.339-44, 1997.

JANTAN, I.; ZAKI, M.Z. Development of environmentfriendly insect repellents from the leaf oils of selected Malaysian plants. Review of Biodiversity and Environmental Conservation, p.1-7, 1998.

LUITGARDS-MOURA, J.F. et al. Preliminary assays indicate that Antonia ovata (Loganiaceae) and Derris amazônica (Papilionaceae), ichthyotoxic plants used for fishing in Roraima, Brazil, have an insecticide effect on Lutzomyia longipalpis (Diptera: Psychodidae: Phlebotominae). Memórias do Instituto Oswaldo Cruz, v.97, p.737-42, 2002.

MARANHÃO, Z.C. Plantas inseticidas. Revista da Agricultura, v.29, p.113-21, 1954.

MATIAS, R. et al. Melia azedarach, uso popular x estudos químicos e farmacológicos: breve revisão. Ensaios e Ciência, v.6, p.91-121, 2002.

MENDONÇA, F.A.C. et al. Activities of some brazilian plants against larvae of the mosquito Aedes aegypti. Fitoterapia, v.76, p.629-36, 2005.

MORAIS, S.M. et al. Larvicidal activity of essential oils from Brazilian croton species against Aedes aegypti L. Journal of the American Mosquito Control Association, v.22, p.161-4, 2006.

MULLA, M.S.; TIANYUN, S. Activity and biological effects of neem products against arthropods of medicinal and veterinary importance. Journal of the American Mosquito Control Association, v.15, n.2,p.133-52, 1999.

MUTHUKRISHMAN, J. et al. Inhibition of juvenile hormone biosynthesis in Gryllus bimaculatus by Glycosmis pentaphylla leaf compounds. Phytochemistry, v.50, p.249-54, 1999.

MUTHUKRISHMAN, J.; PUSHPALATHA, E. Effects of plant extracts on fecundity and fertility of mosquitoes. Journal Applied Entomology, v.125, p.31-5, 2001.

NAKATANI, M. et al. Limonoids from Chukrasia tabularis. Phytochemistry, v.65, p.2833-41, 2004. 
NATHAN, S. S. et al. Efficacy of Melia azedarach L. extract on the malarial vector Anopheles stephensi Liston (Diptera: Culicidae). Bioresource Technology, v.97, p.1316-23, 2005.

NOGUEIRA, M.A.S.; PALMÉRIO, M. Practice oriented results on use and production of plant extracts and pheromones in integrated and biological pest control. In: WORKSHOP NEEM AND PHEROMONES, 1., 2007, Uberaba. Abstracts ... Uberaba: University of Uberaba. Disponível em: <http://www.trifolio-m.de/info_service/ workshops/Brasil_Workshop_1_Abstracts.pdf>. Acesso em: 29 mai. 2007.

PALSSON, K.; JAENSON, T.G.T. Plant products used as mosquito repellents in Guinea Bissau, West Africa. Acta Tropica, v.72, p.39-52, 1999.

PAVELA, R. Insecticidal activity of certain medicinal plants. Fitoterapia, v.75, p.745-9, 2004.

PINTO, A.C. et al. Produtos naturais: atualidade, desafios e perspectivas. Química Nova, v.25, p.45-61, 2002.

ROEL, A.R. Utilização de plantas com propriedades inseticidas: uma contribuição para o desenvolvimento rural sustentável. Revista Internacional de Desenvolvimento Local, v.1, p.43-50, 2001.

SCHLEIN, Y.; JACOBSON, R.L.; MULLER, G.C. Sand fly feeding on noxious plants: a potential method for the control of leishmaniasis. American Journal of Tropical Medicine and Hygiene, v.65, p.300-3, 2001.

SCHMUTTERER, H. Properties and potential of natural pesticides from the neem tree, Azadirachta indica. Annual
Review Entomology, v.35, p.271-97, 1990.

SHAALAN, E.A.S. et al. A review of botanical phytochemicals with mosquitocidal potential. Environment International, v.31, p.1149 -66, 2005.

SILVA, J.J.; MENDES J. Effect of diflubenzuron on stages of Hematobia irritans (L.) (Diptera,Muscidae) in Uberlândia, State of Minas Gerais, Brazil. Memórias do Instituto Oswaldo Cruz, v.97, p.679-82, 2002.

SIMAS, N.K. et al. Produtos naturais para o controle da transmissão da dengue - atividade larvicida de Myroxylon balsamun (óleo vermelho) e de terpenóides e fenilpropanóides. Química nova, v.27, p.46-9, 2004. SIMÕES, C.M.O. et al. Plantas da medicina popular do Rio Grande do Sul. 5.ed. Porto Alegre: UFRGS, 1998. 173p. SIMÕES, C. M.; SPITZER, V. Óleos voláteis. In: SIMÕES, C.M.O. et al. (Eds.). Farmacognosia da planta ao medicamento. 5.ed. Porto Alegre/Florianópolis: UFRS/ UFSC, 2004. 586p

SINNIAH, B.; SINNIAH, D.; IBRAHIM, J. Effect of neem oil and mosquito larvae. Mosquito Borne Disease Bull, v.1, p.90-3, 1994.

VIEGAS-JÚNIOR, C. Terpenos com atividade inseticida: uma alternativa para o controle químico de insetos. Química Nova, v.26, p.390-400, 2003.

WORLD HEALTH ORGANIZATION - WHO. Guidelines for Laboratory and Field Testing of Mosquito larvicides, 2005. Disponível em: http://whqlibdoc.who.int/HQ/2005/ WHO_CDS_WHOPES_GCDPP_2005.13.pdf. 